\title{
Evaluating the Impact of the Electronic Health Record on Patient Flow in a Pediatric Emergency Department
}

DJ Mathison; JM Chamberlain

Children's National Medical Center - Division of Emergency Medicine, Washington, District of Columbia, United States

\section{Keywords}

Emergency medical services, electronic health records, organizational efficiency, workflow, hospital information systems

\section{Summary}

Background: There is little data on the effect of the EHR on emergency department (ED) efficiency. Objective: 1) to quantify the effect of the EHR on patient flow in an academic pediatric ED. 2) to analyze the effects of patient census, boarding time, staffing hours, and acuity on the mean daily ED length-of-stay (LOS) and triage-to-provider time.

Methods: ED performance was compared before and after the implementation of an EHR in May 2008. Six month intervals were used with a 5 month period of adjustment between the pre- and postEHR intervals. 34791 patient visits met inclusion criteria. Multiple linear regression was used to evaluate the LOS and triage-to-provider time as influenced by internal and external variables affecting the ED.

Results: Daily patient census increased by $5.8 \%(p<0.01)$ without a change in rate of ED admissions. Nursing and practitioner hours increased by $19.7 \%$ and $16.1 \%$, respectively because of the increased census and a perceived slowing associated with the EHR. Following the implementation, LOS remained unchanged while triage-to-provider time increased by 5 minutes per patient $(p<0.05)$. Factors that independently affected both LOS and triage-to-provider time included census, acuity, and practitioner hours $(p<0.05)$. When controlling for these independent variables, the use of an EHR did not affect either outcome variable $(p=0.251,0.074$ respectively). However, patient flow was worsened with the EHR during days of extremely high patient census.

Conclusion: An ED-EHR was associated with a modest increase in time to see a medical provider but was not associated with a change in overall LOS. When controlling for factors including patient volume, acuity, and staffing, the EHR did not independently affect ED patient flow. The EHR may have a more profound impact on ED performance during periods of extremely high census.

\section{Correspondence to:}

Children's National Medical Center - Division of Emergency Medicine

111 Michigan Ave. NW DC Washington 20010

United States

Phone: 2024764177

E-mail: davidmathison@hotmail.com
Appl Clin Inf 2011; 2: 39-49

doi: 10.4338/ACl-2010-08-RA-0046

received: August 12, 2010

accepted: January 22, 2011

published: February 2, 2011

Citation: Mathison DJ; Chamberlain JM.: HIS-based support of follow-up documentation - concept and implementation for clinical studies. Appl Clin Inf 2011; 2: $39-49$

http://dx.doi.org/10.4338/ACI-2010-08-RA-0046 


\section{Background}

As both the demand and cost for quality health care services rise, increasing attention has been directed towards health information technology (HIT) as a solution to improve the efficiency, quality, and safety of medical care [1]. The electronic health record (EHR) is one of the core HIT solutions. The introduction of an EHR can improve quality of care [2-4], financial performance [3, 57], and patient outcome [8]. In the U.S., recent legislation has mandated that all U.S. health records be computerized by 2014 [9].

Some of the benefits of EHRs, however, are countered by the effects of altered physician workflows and a change in the way physicians document their patient encounters [10]. Computerized documentation in EHRs, in particular, can cause worsening time efficiency among physicians and nurses [8], thus resulting in longer patient cycle times and increased patient lengths-of-stay (LOS) in ambulatory settings [6]. These decreased efficiencies can be particularly detrimental in the fastpaced setting of emergency departments (EDs), where workflow interruptions are commonplace. As ED overcrowding becomes increasingly prevalent [11-13], hospital EDs search for methods to improve workflow efficiency, often measured by patient LOS and the time from triage-to-provider. While computerized physician order entry (CPOE) has been associated with a decrease in ED LOS [7], the EHR (with CPOE) may worsen ED LOS [14]. No study to-date has evaluated the impact of the EHR (with CPOE) on ED efficiency while accounting for variations in ED inputs, staffing adjustments, and the change management that naturally occurs with HIT implementation.

\section{Objectives}

This study evaluates the effects of the EHR with CPOE on patient flow in an academic ED setting, specifically addressing the impact of patient census, boarding time, staffing hours, and patient acuity on the mean daily triage-to-provider time and ED length-of-stay (LOS).

\section{Methods}

\subsection{Study Design}

This was a retrospective analysis that compared data before and after the implementation of an EHR in May 2008. Six month data-intervals were used: October 1, 2007-March 31, 2008 and October 1, 2008-March 31, 2009. This allowed a 5-month period of adjustment to exclude the shortterm effects of change management that naturally accompany a technology transformation. Prior to the implementation of the EHR, all ED physician and ED nursing documentation was recorded on paper. Physicians placed all orders on the paper charts. Nurses transcribed the laboratory and radiology orders into a computerized order entry system and faxed or tubed medication orders (not available in the ED) to pharmacy. Electronic patient tracking and patient discharge instructions (LogiCare Corporation, Eau Claire, WI), were used. The implementation of the EHR allowed a movement to complete electronic (typed) documentation by both physicians and nurses. Physicians entered all orders using computerized physician order entry (CPOE) with decision support. Additionally, patient tracking was transitioned from the old vendor to the new vendor (Cerner, Inc., Kansas City, MO).

\subsection{Study Setting}

Children's National Medical Center (CNMC) is a large, urban, tertiary care, academic pediatric facility with an ED that sees approximately 85,000 visits per year. This level 1 pediatric trauma center is a major referral center for the mid-Atlantic region. The ED staffing model utilizes 19-22 daily providers who compose a relatively constant mix of pediatric emergency physician attendings, pediatric emergency physician fellows, nurse practitioners, pediatric associates (e.g., pediatricians), 
and medical residents. At any given time there are 4-12 providers and 12-19 registered nurses (RNs), fluctuating by the anticipated volumes at a particular time of day. Resident physicians are a mix of in-house pediatrics and rotating residents from either pediatric, emergency medicine, or family medicine training programs. The composite of resident providers is relatively consistent with little day-to-day variability.

\subsection{Study Protocol}

Data sets contained time events to the nearest minute for arrival, triage, initial provider examination, admission order, discharge order, and discharge from ED (disposition). > Figure 1 shows these relative time events. Triage time, provider sees time, and disposition time were entered manually by nurses and physicians on the tracking board during the course of normal workflow. Each of these times represented the initiation of activity, such as nurse triage. Arrival time was recorded automatically when the patient began the registration process, requiring only name and date of birth. Disposition time was the time event for removing the patient from the tracking board which occurred just following the patient leaving the physical bed space (for discharge or admission). The LOS was calculated for each patient subtracting the initial arrival time from the disposition time. The triage-to-provider time was calculated for each patient by subtracting the triage time from the provider sees time. The definition of a provider sees event was the patient's first interaction with any medical provider, which could include a resident physician, fellow physician, attending physician, or a nurse practitioner. Inpatient boarding time was calculated by subtracting the admission order time from the disposition time. Boarding time measured the time that patients waited before being admitted to an inpatient bed. Boarding time was interpreted as a downstream gauge of hospital census which impacts ED patient flow regardless of a patient's disposition. Mean and median daily data for each outcome were determined by using all patients with ED arrivals within each 24hour period from 12:00 am to 11:59 pm.

The implementation of the EHR was expected to require a larger staffing force both in the shortand long-term to accommodate for the process change. Thus, both nursing and provider staffing were built into this model. The daily nursing hours and provider hours were recorded from schedule software (Suite Scheduler, Eau Claire, WI). These data included additional staffing hours required during periods of increased census and excluded the hours of the staff who were sick and did not work the shift scheduled. The staffing did not include resident physicians, who were consistently staffed and did not fluctuate during periods of low and high census or between the pre- and post-EHR periods.

Daily acuity level was defined as the total number of admissions, deaths, and outgoing transfers per day. While a triage acuity level (1-5) is recorded for every patient, we have noted historically that triage acuity level is a better determinant of urgency while admission rate is a better determinant of the true overall acuity in the ED. At our facility, a high triage acuity level requires rapid therapy (such as asthma therapy or narcotic pain control) but does not consistently correlate with LOS or the time physicians spend with or make decisions about their patients. When calculating a daily acuity level, we did not weight admissions by destination such as ICU versus regular ward. We excluded the following patients from analysis:

1. Patients triaged to a low-acuity unit called fast track (urgent care) were excluded because the EHR was not implemented at this time for these patients and time data were not consistently recorded;

2. Patients admitted to the hospital were excluded to remove outliers that stayed in the ED for prolonged periods of time awaiting either an inpatient bed or transfer to another facility. At CNMC, the LOS for admitted patients is unreliable secondary to the wide variability in boarding time for patients depending on the fluctuating bed availabilities of different subspecialty services. Additionally, the time to admission order was not consistently documented in the pre-EHR workflow.

3. Patients transferred from another hospital were excluded because their arrival time (i.e. registration time) occurred prior to actual arrival at the facility; and

4. Patients with inaccurate or incomplete time event data related to resuscitation, registration errors, missing events, or negative time intervals. 
The resulting data set consisted of largely mid-level acuity patients who were discharged home from the ED.

\subsection{Measures \& Analysis}

The primary dependent variables were mean daily LOS and mean daily triage-to-provider time. These variables were analyzed with general linear multivariate regression with respect to the independent variables, which included daily census, mean daily boarding time, daily acuity, daily nursing staffing hours, and daily provider staffing hours. The continuous dependent and independent variables were evaluated for significance using bivariate analyses (t-tests). SPSS (v17.0, SPSS, Inc., Chicago, IL) was used for all statistical analysis.

The study was approved by the Institutional Review Board (IRB) for human subject research and data collection was compliant with the Health Insurance Portability and Accountability Act of 1996.

\section{Results}

The sample size was 365 days (183 pre-EHR and 182 post-EHR). This sample set included 34,791 unique patient visits (16,951 pre-EHR and 17,840 post-EHR). $>$ Figure 2 depicts patient inclusion and exclusion. The period after implementation saw an increase in daily patient census by $5.8 \%$ (228.5 \pm 28.6 to $241.7 \pm 31.9, p<0.001$ ) with only a marginal change in the proportion of ED patients hospitalized $(12.03 \% \pm 2.50 \%$ to $11.84 \% \pm 2.02 \%, p<0.001)$. Nursing and medical practitioner hours increased by $19.7 \%$ and $16.1 \%$, respectively because of the increased census and a perceived slowing associated with the EHR ( $>$ Table 1$)$. Boarding time decreased $(p<0.001)$ related to process improvements with admissions and inpatient bed availability. The post-EHR period had relatively more patients excluded as "other disposition" and "time entry errors" secondary to changes in the registration process for transferred patients and mistakes made removing patients from the tracking board. These excluded patients accounted for a marginal percentage of this overall analysis and likely had an insignificant impact on ED patient flow.

Mean daily triage-to-provider time increased after the implementation of the EHR $(76.5 \pm 21.2$ versus $81.5 \pm 24.2$ minutes, $p<0.037)$. Mean LOS was not affected $(234.3 \pm 29.1$ versus $235.8 \pm 28.8$ minutes, $\mathrm{p}=0.63$ ).

When controlling for the improved boarding times, increased staffing, and increased patient census in the post-implementation period, the utilization of the EHR did not have a significant independent effect on either the mean daily ED LOS or the mean daily triage-to-provider time ( Table 2). Only patient census, mean daily acuity, and provider staffing had significant independent effects on both mean LOS and mean triage-to-provider time.

- Figure 3 depicts the flow outcomes of triage-to-provider time and ED LOS as a function on total daily patient volume. In the post-EHR period, the curve shows a lower baseline but a steeper slope and it crosses the curve for the pre-EHR period during higher census. This suggests that the baseline increase in staffing predominates during periods of low census but the inefficiencies of the EHR predominate during periods of high census.

\section{Discussion}

To our knowledge, this is the first description of the adjusted effect of an EHR (with CPOE) on patient flow in a large academic ED setting. Despite the perceived notion that the EHR has slowed patient flow, we showed that after allowing a five-month short-term period of adjustment and controlling for other associated variables, the use of the EHR did not have a significant impact on either LOS or Triage-to-Provider time for the average mid-level acuity discharged patient. The implementation of the EHR did require approximately $16 \%$ more medical provider hours to see an increase in patient volume of approximately $6 \%$, suggesting inefficiencies that could not be measured using our methodology. Furthermore, the curves for waiting times as a function of total daily volumes 
were steeper in the post-EHR period, suggesting that flow was more sensitive to volume after implementation of the EHR.

Our findings are in contrast to those by Vartak et al. [14], who found that the implementation of a multi-system solution consisting of an EHR, CPOE, Clinical Decision Support (DSS), and electronic patient tracking caused an increase in the mean LOS by 17 min (approximately 15\% per patient). This study incorporated a time period immediately after the implementation, was in the setting of decreasing ED volumes, and did not control for other changes or process improvements such as a reorientation of their triage process that occurred with the implementation. In addition, our staff was already familiar with the use of electronic patient tracking and the use of electronically generated discharge instructions. This may have allowed an easier transition to the EHR and simultaneously allowed us to study the EHR impact in isolation.

Daniel et al. [7] found a favorable effect of an EHR on ED flow, however this payer-based health technology provided only improved patient health information (patient medical history) to physicians and did not alter ED workflow with additional or changed steps such as physician charting, electronic tracking, or CPOE. This study does, however, speak to the potential benefits of EHR's and the ability for ED physicians to work more efficiently when health information is more readily accessible.

Our data was similar to but more linearly distributed (less logarithmic) than a previous analysis of patient flow dynamics at a comparably-sized pediatric academic institution as described by Timm et al. [15].

While several studies have examined the impact of HIT solutions such as DSS on quality improvement or physician performance, few studies have examined the EHR in isolation or in a highvolume clinical setting such as an urban, academic ED. Hospital EDs that are considering implementation of EHRs should prepare for the short-term inefficiencies of change management, but should not require long-term alterations in patient flow based on the EHR alone. Patient flow may be more sensitive to high patient volumes after implementation of the EHR. Further research is needed to determine if alternative charting, such as using paper, would be an advantageous strategy to improve patient flow during periods of high patient census. Such a system would, however, carry inherent inefficiencies and risks during the transition periods.

This study has some limitations. We excluded the extreme patient groups, both the large percentage of low-acuity visits and the small percentage of resuscitations that did not use the EHR, ultimately measuring the mid-level non-admitted patient. This group represents the 'core' of the ED and effects on this group could be extrapolated to the ED as a whole. Since this study measured flow in a high-volume high-acuity center and excluded low-acuity visits, it is possible that the EHR may have a more pronounced effect on urgent care centers or EDs with greater percentages of low acuity visits. Admission times were not used as an outcome because admission times, unlike LOS for discharged patients, are often beyond the locus of control of the ED. Future studies could incorporate the low-acuity patients who arguably are even more affected by the overcrowding phenomenon and longer lengths of stay. Also, time event data were not tracked passively, so there is the possibility for occasional errors in recording time events. Our practice is to record patient activities in real time and to modify the time stamp post hoc when real-time event recording is not possible (e.g. because of immediate need for resuscitation). There is no reason to suspect a systematic bias in recording time events between the two study periods. For medical trauma and resuscitations, our institution continues to use paper charting. These events inevitably slow patient flow in the ED and conversion to an EHR for resuscitations has yet to be implemented at our institution which may speak to the difficulty of incorporating live time event tracking during hyperacute situations.

\section{Conclusions}

The period after the implementation of an ED-based EHR was associated with a modest increase in time to see a medical provider but was not associated with a change in overall LOS.

Daily provider staffing, patient census, and patient acuity were strongly associated with patient flow before and after EHR implementation. When controlling for these factors, the EHR did not independently affect patient flow. The results of this study highlight the misperceptions commonly 
associated with EHRs. While nursing and physician documentation time was likely worsened by the use of an EHR, these time efficiencies did not impact the total ED LOS for mid-level acuity patients.

\subsection{Implications}

For hospitals considering EHR implementation, special attention should be paid to improving provider staffing which in this study contributed to improved ED efficiency and, unlike patient census or acuity, can be easily anticipated and modified. We speculate that our increase in nurse and provider staffing likely contributed to improved efficiencies not well represented in this model. Since the effect of the EHR may be more pronounced during periods of high patient census, hospitals could consider alternative documentation during overcrowding situations such as mass casualty scenarios, high occupancy alerts, or periods of ambulance diversion. It is unclear if these principles can be extrapolated to other acute care settings since the ED is a highly specialized and unique team-oriented patient care environment. Future studies are needed to assess the impact of the EHR/CPOE on low acuity patients and potential efficiency solutions during periods of high patient census.

\section{Conflicts:}

The authors declare they have no conflicts of interest in this research. 


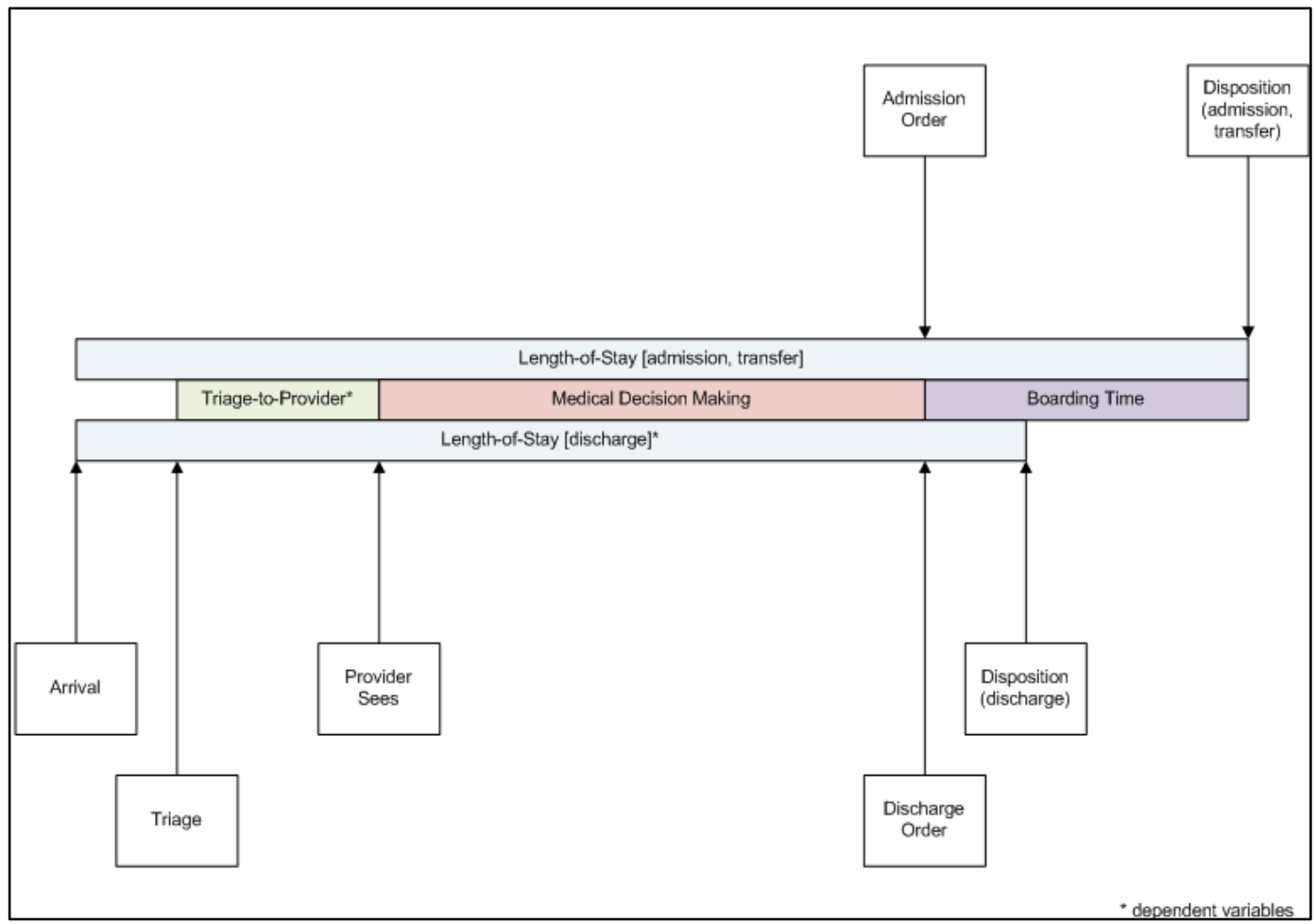

Fig. 1 Timeline of ED patient flow 


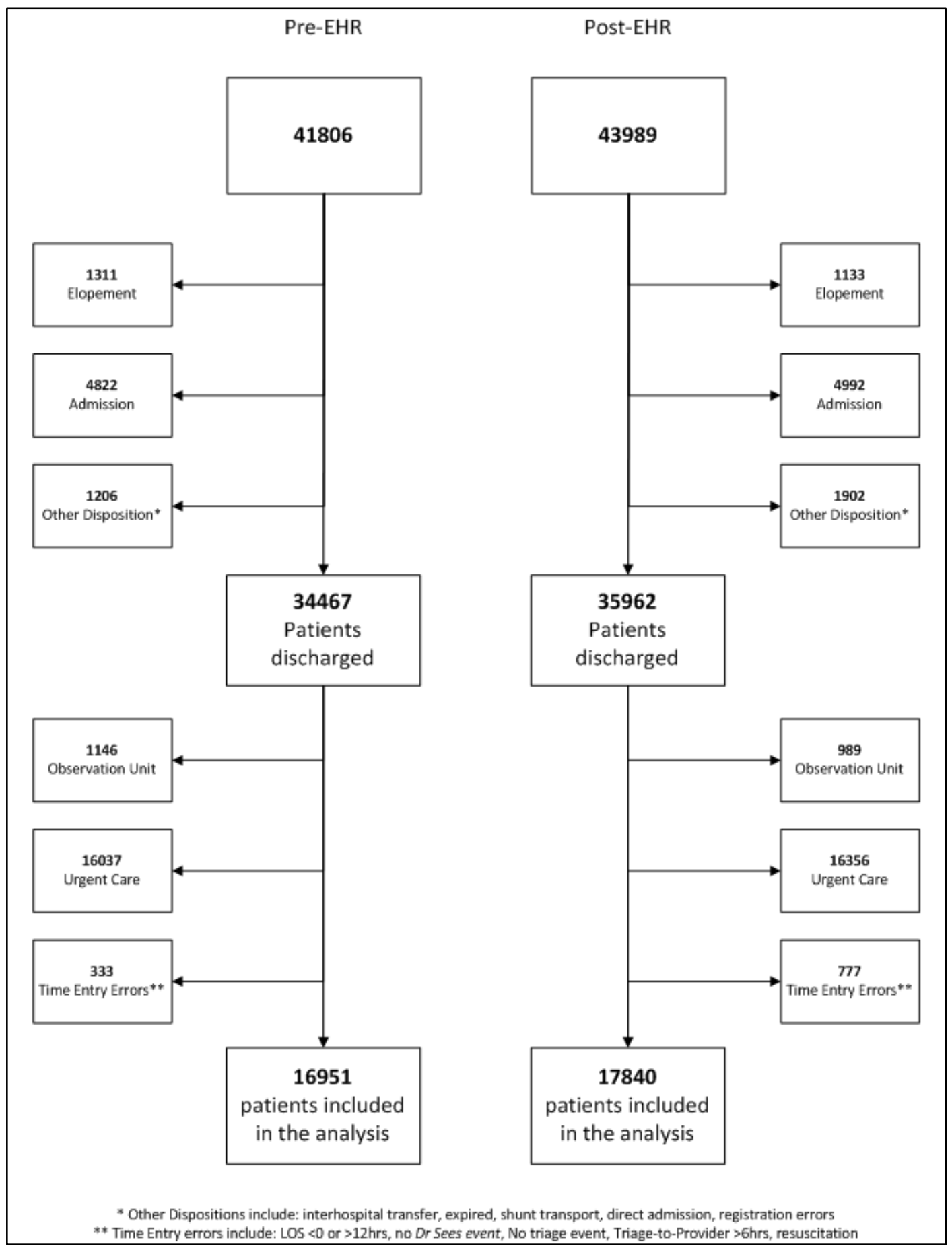

Fig. 2 Patient recruitment and exclusion 


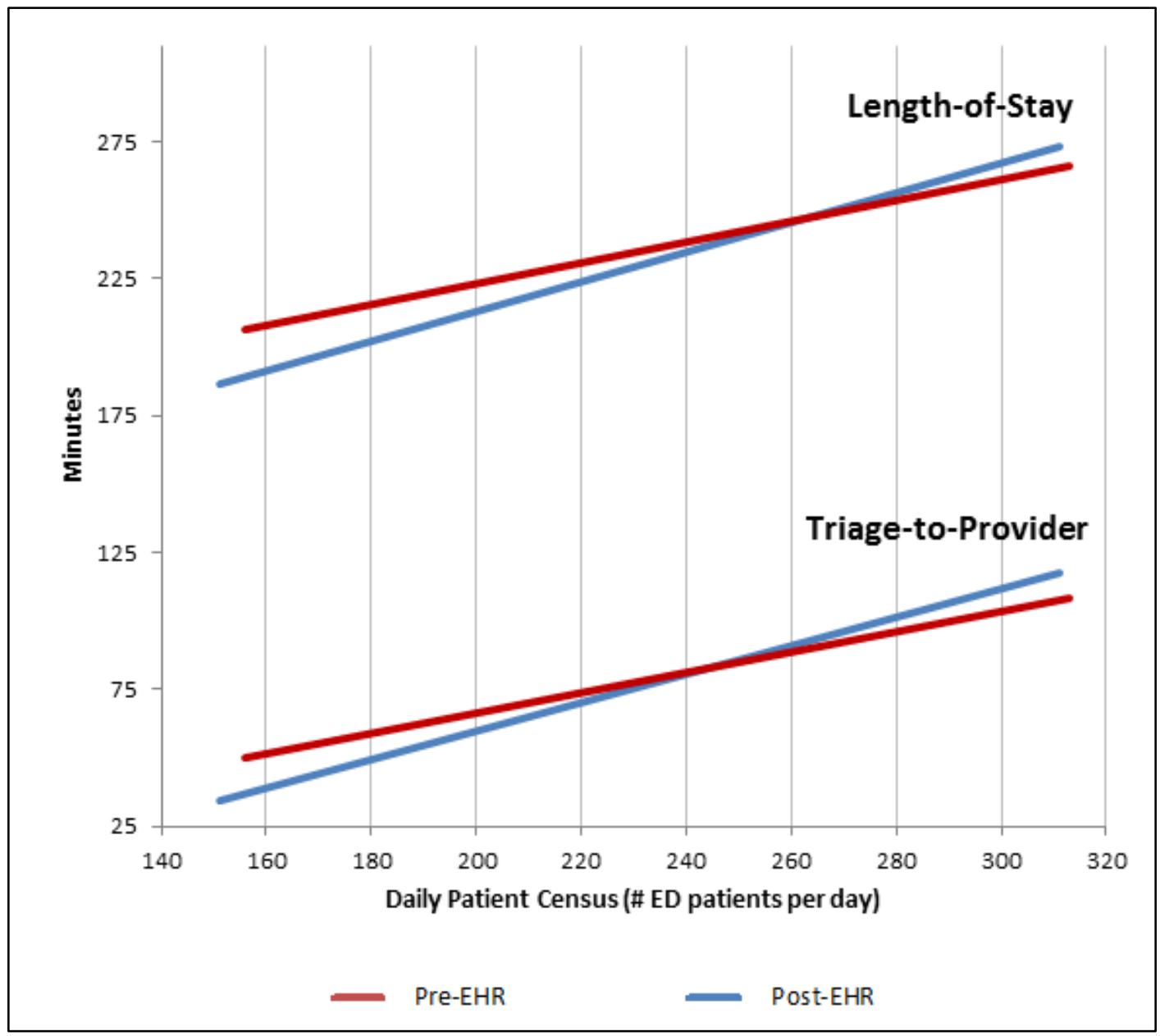

Fig. 3 Mean daily LOS and triage-to-provider time vs. daily ED patient census 
Table 1 Comparison of Results

\begin{tabular}{|l|l|l|l|}
\hline Daily Totals & PRE-EHR & POST-EHR & P-value \\
\hline & $\mathrm{N}=183$ days & $\mathrm{N}=182$ days & \\
\hline Mean LOS & $234.3 \mathrm{~min}( \pm 29.1)$ & $235.8 \mathrm{~min}( \pm 28.8)$ & 0.630 \\
\hline Mean Triage-to-Provider Time & $76.5 \mathrm{~min}( \pm 21.2)$ & $81.5 \mathrm{~min}( \pm 24.2)$ & 0.037 \\
\hline Mean Daily Patient Census & 228 patients $( \pm 28.6)$ & 241.7 patients $( \pm 31.9)$ & 0.001 \\
\hline Mean Daily Practitioner Hours & 139.7 hours $( \pm 19.2)$ & 162.2 hours $( \pm 17.8)$ & 0.001 \\
\hline Mean Daily Nursing Hours & 267.9 hours $( \pm 19.7)$ & 320.8 hours $( \pm 23.5)$ & 0.001 \\
\hline Mean Daily Acuity* & $27.5( \pm 5.6)$ & $28.6( \pm 6.0)$ & 0.070 \\
\hline Mean Boarding Time & 145.9 min $( \pm 20.3)$ & 126.6 min $( \pm 20.3)$ & 0.001 \\
\hline
\end{tabular}

Table 2 Multivariate Regression Analysis

\begin{tabular}{|c|c|c|c|c|c|c|}
\hline & \multicolumn{3}{|c|}{ Mean Daily LOS } & \multicolumn{3}{|c|}{$\begin{array}{l}\text { Mean Daily Triage-to-Provider } \\
\text { Time }\end{array}$} \\
\hline & \multicolumn{3}{|c|}{$R^{2}=0.339$} & \multicolumn{3}{|c|}{$R^{2}=0.489$} \\
\hline & Beta & $\mathrm{Cl}$ & $p$-value & Beta & $\mathrm{Cl}$ & $p$-value \\
\hline Patient Census & 0.42 & $0.33-0.52$ & 0.000 & 0.44 & $0.37-0.51$ & 0.000 \\
\hline Acuity (Transfer/Admit/Death) & 1.26 & $0.79-1.74$ & 0.000 & 1.04 & $0.71-1.37$ & 0.000 \\
\hline Boarding Time & 0.16 & $0.04-0.28$ & 0.012 & 0.07 & $-0.02-0.16$ & 0.111 \\
\hline Nursing Hours & -0.03 & $-0.15-0.89$ & 0.614 & 0.03 & $-0.06-0.11$ & 0.531 \\
\hline Provider Hours & -0.26 & $-0.41-(-) 0.10$ & 0.001 & -0.32 & $-0.43-(-) 0.22$ & 0.000 \\
\hline Electronic Health Record & 4.85 & $-3.44-13.15$ & 0.251 & 5.24 & $-0.51-11.00$ & 0.074 \\
\hline
\end{tabular}




\section{References}

1. Jamal A, McKenzie K, Clark M. The impact of health information technology on the quality of medical and health care: a systematic review. HIM J 2009; 38(3): 26-37. PMid:19875852

2. Adams WG, Mann AM, Bauchner H. Use of an electronic medical record improves the quality of urban pediatric perimary care. Pediatrics. 2003;111(3):626-632. doi:10.1542/peds.111.3.626 PMid:12612247

3. Shekelle PG, Morton SC, Keeler EB. Costs and benefits of health information technology. Evid Rep Technol Assess 2006; 132: 1-71.

4. Chaudhry B, Wang J, Wu S, Maglione M, Mojica W, Roth E, et al. Systematic review: impact of health information technology on quality, efficiency, and costs of medical care. Ann Intern Med 2006; 144(10): 742-752. PMid:16702590

5. Zaroukian $\mathrm{MH}$, Sierra A. Benefiting from ambulatory EHR implementation: solidarity, six sigma, and willingness to strive. J Healthc Inf Manag 2006; 1: 53-60.

6. Samaan ZM, Klein MD, Mansour ME, DeWitt TG. The impact of the electronic health record on an academic pediatric primary care center. J Ambul Care Manage 2009; 32(3): 180-187. PMid:19542807

7. Spalding SC, Mayer PH, Ginde AA, Lowenstein SR, Yaron M. Impact of computerized physician order entry on ED patient length of stay. Am J Emerg Med. 2010.

8. Poissant L, Pereira J, Tamblyn R, Kawasumi Y. The impact of electronic health records on time efficiency of physicians and nurses: a systematic review. J Am Med Inform Assoc 2005; 12(5): 505-516. doi:10.1197/jamia.M1700 PMid:15905487 PMCid:1205599

9. $111^{\text {th }}$ U.S. Congress. American Recovery and Reinvestment Act of 2009. Title XXX - Health Information Technology and Quality. In: http://frwebgate.access.gpo.gov/cgibin/getdoc.cgi?dbname=111_cong_bills\&docid=f:h1enr.pdf.

10. Irani JS, Middleton JL, Marfatia R, Omana ET, D'Amico F. The use of electronic health records in the exam room and patient satisfaction: a systematic review. J Am Board Fam Med 2009; 22(5): 553-562. doi:10.3122/jabfm.2009.05.080259 PMid:19734402

11. Derlet RW, Richards JR. Overcrowding in the nation's emergency departments: complex causes and disturbing effects. Ann Emerg Med 2000; 35(1): 63-68. doi:10.1016/S0196-0644(00)70105-3

12. Hostetler MA, Mace S, Brown K, Finkler J, Hernandez D, Krug SE, et al. Emergency department overcrowding and children. Pediatr Emerg Care 2007; 23(7): 507-515. doi:10.1097/01.pec.0000280518.36408.74 PMid:17666940

13. Trzeciak S, Rivers EP. Emergency department overcrowding in the United States: an emerging threat to patient safety and public health. Emerg Med J 2003; 20(5): 402-405. doi:10.1136/emj.20.5.402 PMid:12954674 PMCid:1726173

14. Vartak S, Crandall DK, Brokel JM, Wakefield DS, Ward MM. Transformation of emergency department processes of care with EHR, CPOE, and ER event tracking systems. HIM J 2009; 38(2): 27-32. PMid:19546485

15. Timm NL, Ho ML, Luria JW. Pediatric emergency department overcrowding and impact on patient flow outcomes. Acad Emerg Med 2008; 15: 832-837. doi:10.1111/j.1553-2712.2008.00224.x PMid:18821860 